\title{
EchoGéo
}

33 | 2015

Activisme, participation, contestation : la place des habitants dans les processus de patrimonialisation en périphéries urbaines

\section{Prise en compte de la pluralité des mémoires d'habitants dans la « patrimonialisation » des grands ensembles}

Réflexion à partir du cas stéphanois

\section{Rachid Kaddour}

\section{(2) OpenEdition} Journals

Electronic version

URL: https://journals.openedition.org/echogeo/14337

DOI: $10.4000 /$ echogeo. 14337

ISSN: 1963-1197

Publisher

Pôle de recherche pour l'organisation et la diffusion de l'information géographique (CNRS UMR 8586)

\section{Electronic reference}

Rachid Kaddour, "Prise en compte de la pluralité des mémoires d'habitants dans la "patrimonialisation » des grands ensembles", EchoGéo [Online], 33 | 2015, Online since 30 September 2015, connection on 11 August 2021. URL: http://journals.openedition.org/echogeo/14337 ; DOl: https://doi.org/10.4000/echogeo.14337

This text was automatically generated on 11 August 2021.

EchoGéo est mis à disposition selon les termes de la licence Creative Commons Attribution - Pas d'Utilisation Commerciale - Pas de Modification 4.0 International (CC BY-NC-ND) 


\section{Prise en compte de la pluralité des mémoires d'habitants dans la « patrimonialisation » des grands ensembles}

Réflexion à partir du cas stéphanois

Rachid Kaddour

1 En ce milieu de décennie, le grand ensemble de Beaulieu à Saint-Etienne fête coup sur coup deux anniversaires symboliques : 2015 est l'année du soixantenaire de sa livraison, alors qu'avant cela 2013 correspond aux dix ans de l'obtention de son label Patrimoine $d u X X^{e}$ siècle. Ce label inscrit Beaulieu dans un processus de valorisation patrimoniale par l'architecture, et le place ainsi en terrain laboratoire pour une réflexion sur la patrimonialisation des grands ensembles (Pouvreau, 2011 ; Kaddour, 2013).

2 Cette labellisation n'est toutefois pas la seule démarche de valorisation patrimoniale et mémorielle conduite pour Beaulieu et les grands ensembles voisins : les années 2000 voient s'y multiplier les entreprises visant à identifier et valoriser d'une part les singularités architecturales et urbaines de Beaulieu, et d'autre part les récits de vie contrastés de différents groupes d'habitants des grands ensembles. La somme de ces entreprises patrimoniales et mémorielles, près d'une dizaine, conforte l'idée d'exemplarité des Quartiers sud-est de Saint-Étienne. D'autant plus que, dans l'histoire des grands ensembles français, ces Quartiers sont particulièrement précurseurs: Beaulieu est l'un des plus vieux de France, tandis que son voisin Montchovet est un pionnier de la politique de la Ville, puis de la rénovation urbaine et des travaux de deuil par le recueil de mémoires qui accompagnent cette dernière.

3 Si l'on se place dans une perspective d'analyse des jeux d'acteurs, représentations et stratégies conditionnant les processus de fabrication du patrimoine, suivant en cela les approches traditionnellement menées par la géographie sociale (Gravari-Barbas, 2002 ; Veschambre, 2007; Hertzog, 2011), il est possible de s'interroger ici plus spécifiquement sur la place laissés aux mémoires plurielles d'habitants dans la 
définition et la valorisation de ce qui fait patrimoine dans les grands ensembles du sudest de Saint-Étienne. La particularité de cette réflexion réside dans le fait de la placer au cœur de la rénovation urbaine qui fait l'actualité des grands ensembles.

4 Les informations analysées ici ont été recueillies par une recherche documentaire (revue de presse, consultation d'archives administratives et de documents techniques), par la réalisation d'entretiens avec des représentants des différentes institutions et associations impliquées (directeurs successifs de l'organisme d'HLM, techniciens du service Urbanisme de la Ville, travailleurs sociaux et enseignants) ainsi qu'avec des habitants, et par des observations sur site (transformations spatiales).

Un retour sur la livraison et l'évolution des Quartiers sud-est permettra dans un premier temps de donner la contextualisation nécessaire à la compréhension de ce qui se joue aujourd'hui. Cet historique permettra d'insérer ensuite dans l'épaisseur et la dynamique du temps long un inventaire espéré exhaustif des initiatives réalisées depuis le lancement de la rénovation urbaine par les différents acteurs institutionnels et associatifs. Cet inventaire est réalisé à partir d'une définition large de la notion de patrimoine, prise dans ses différentes acceptions notariale, immobilière et culturelle. Ces éléments contextuels et factuels collectés, il s'agira enfin, dans le dernier tiers de cette présentation, de les confronter et de les mettre en cohérence afin de proposer une interprétation sur ce qui fait patrimoine dans les grands ensembles du sud-est de SaintEtienne. Cela permettra une réflexion sur la place que les mémoires plurielles d'habitants y tiennent.

\section{Quartiers sud-est de Saint-Étienne : des grands ensembles contrastés, des habitants divers}

6 Cette première partie a pour objet de présenter les grands ensembles du sud-est de Saint-Étienne. Pour cela, il est nécessaire de prendre le temps d'en faire un historique. Il s'agit notamment, en plus de donner les éléments urbains et économiques propres à l'agglomération stéphanoise dans la grande région lyonnaise, de s'intéresser aux acteurs de la vie de ces grands ensembles depuis leur construction, en présentant les principales actions menées par les institutions gestionnaires, et en identifiant les principales catégories sociales d'habitants ayant successivement occupé les lieux. Au niveau des sources, cet historique s'appuie sur les travaux existants, complétés par des études techniques (dont le dossier de candidature au Grand projet de ville - GPV), une revue de presse et des entretiens avec les gestionnaires.

$7 \mathrm{Au}$ lendemain de la Seconde Guerre mondiale, la ville de Saint-Étienne se voit surnommée Capitale des taudis ${ }^{1}$ en raison des taux élevés de surpeuplement et d'insalubrité de son parc de logements. Cette crise du logement débute avec l'important développement industriel du XIXe siècle et la fulgurante croissance démographique qui l'accompagne: de 25000 personnes après la Révolution française, la population stéphanoise passe à plus de 190000 dans les années 1930. Or, la construction d'habitations par le patronat ne suit pas, les compagnies minières et métallurgiques se contentant de laisser les ouvriers se loger dans l'existant et les immeubles des artisans. Comme dans nombre de villes françaises, c'est la construction de grands ensembles au début des années 1950 qui permet de porter un coup décisif à cette crise tenace. La ville présente la particularité d'abriter l'un des six premiers grands ensembles de France: imaginé à partir de 1949, Beaulieu est construit entre 1953 et 1955 dans le cadre du plan 
national dit Courant ${ }^{2}$, et compte 1262 logements HLM modernes et confortables. Il n'est que la première opération d'un plan plus vaste qui, pour ce qui concerne le seul sud-est de la ville, amène à la production de plus de 6000 logements, dans les grands ensembles de Beaulieu II - Marandinière (1957-1959), Beaulieu III - Montchovet (1962-1965), Beaulieu IV - la Palle (1967-1970) et dans celui de la Métare en copropriété (1962-1974, voir illustration 1).

8 C'est plus particulièrement sur les grands ensembles de Beaulieu et Montchovet, mitoyens, que l'attention va ici porter. Leurs formes divergent, puisque le premier compte 19 barres et une tour formant un ensemble urbain particulièrement soigné (illustration 2), alors que le second (1 266 logements) est composé de huit barres, certaines très imposantes, implantées simplement parallèlement ou perpendiculairement les unes aux autres.

Illustration 1 - Les Quartiers sud-est à leur livraison

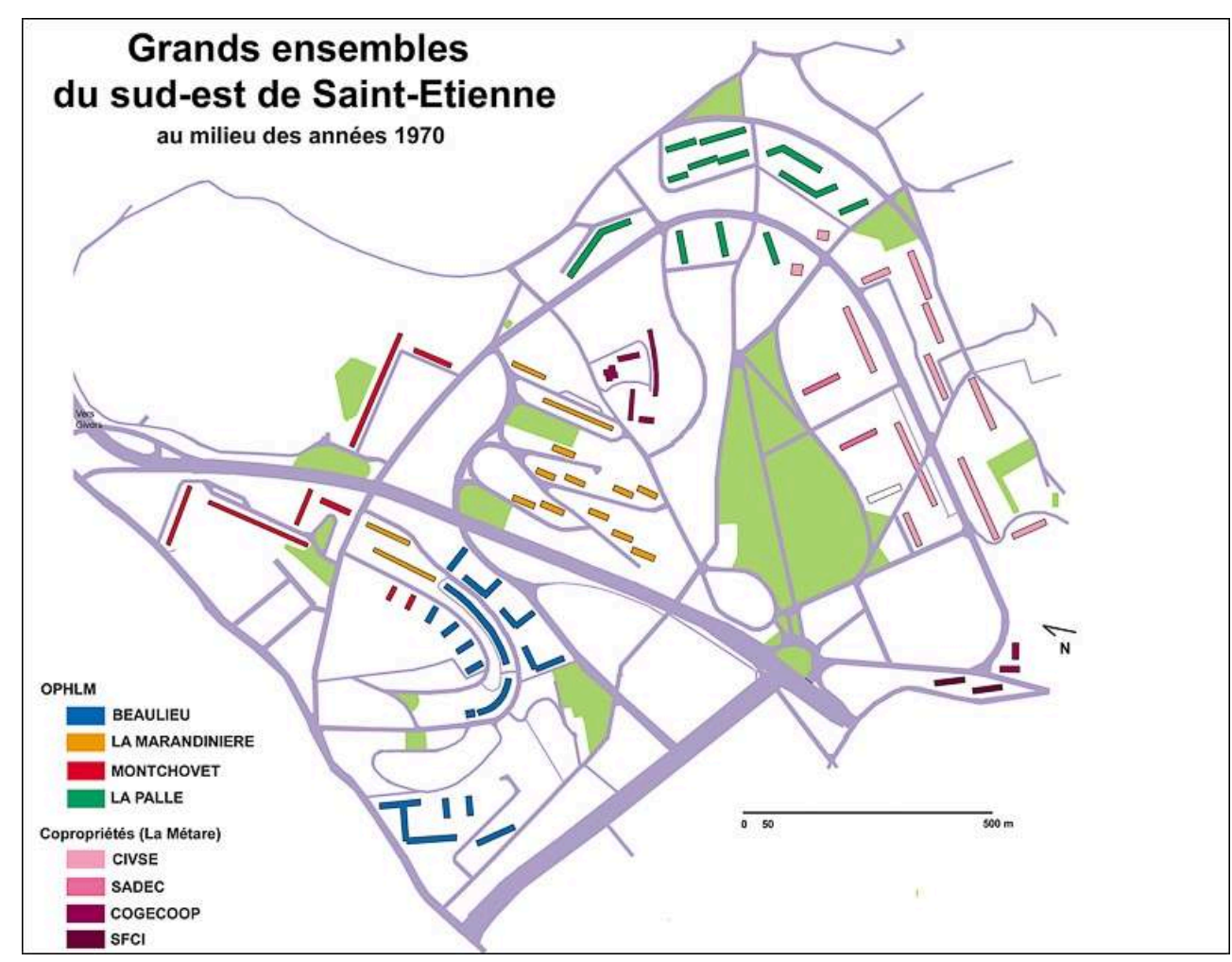

Auteur: R. Kaddour. 
Illustration 2 - Vue aérienne de Beaulieu à sa livraison

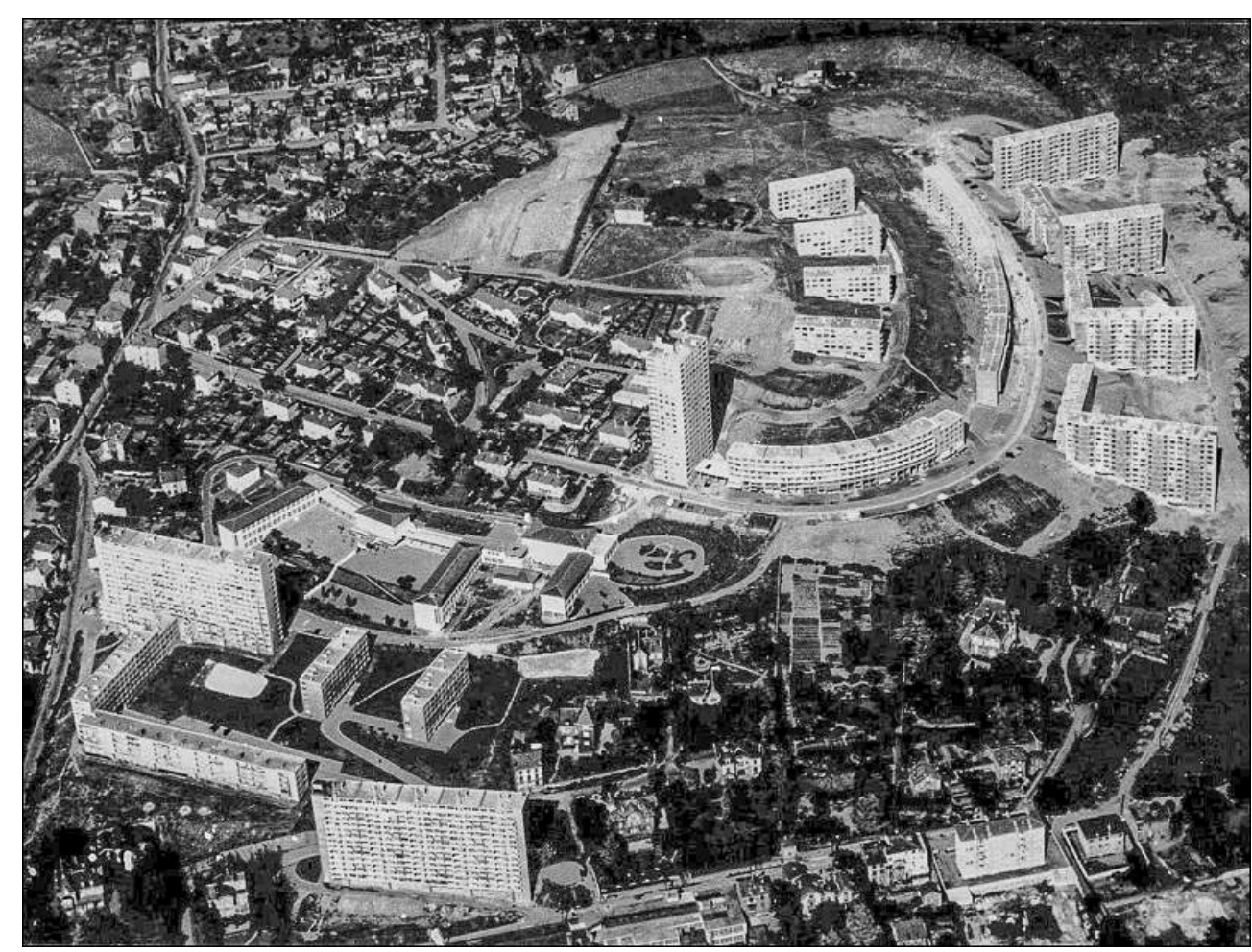

La composition organique de l'ensemble est très repérable sur la droite de l'image.

Source : Archives municipales de Saint-Étienne.

9 Leurs occupations initiales (Vant, 1974 ; Blanc, Bonilla, Tomas, 2003) sont par contre significativement proches, puisqu'ils accueillent tous deux très majoritairement des ouvriers de l'industrie (principalement qualifiés) et des fonctionnaires, soit des catégories sociales que l'on qualifierait aujourd'hui de moyennes (illustration 3) 3. La $^{3}$ crise du logement est en effet telle à Saint-Étienne que les demandes d'HLM émanent de catégories sociales très variées, et, pour les grands ensembles, l'office municipal gestionnaire sélectionne les locataires suivant leur solvabilité. Les plus pauvres des demandeurs de logements HLM, ainsi que les immigrés et familles originaires du Maghreb, quand ils sont accueillis, le sont dans des opérations de taille plus réduite, formant un habitat spécifique (Logements de première nécessité, Programmes sociaux de relogement, opération Million...). Autrement, ils occupent le parc de logements vieilli, insalubre ou précaire de la ville. 


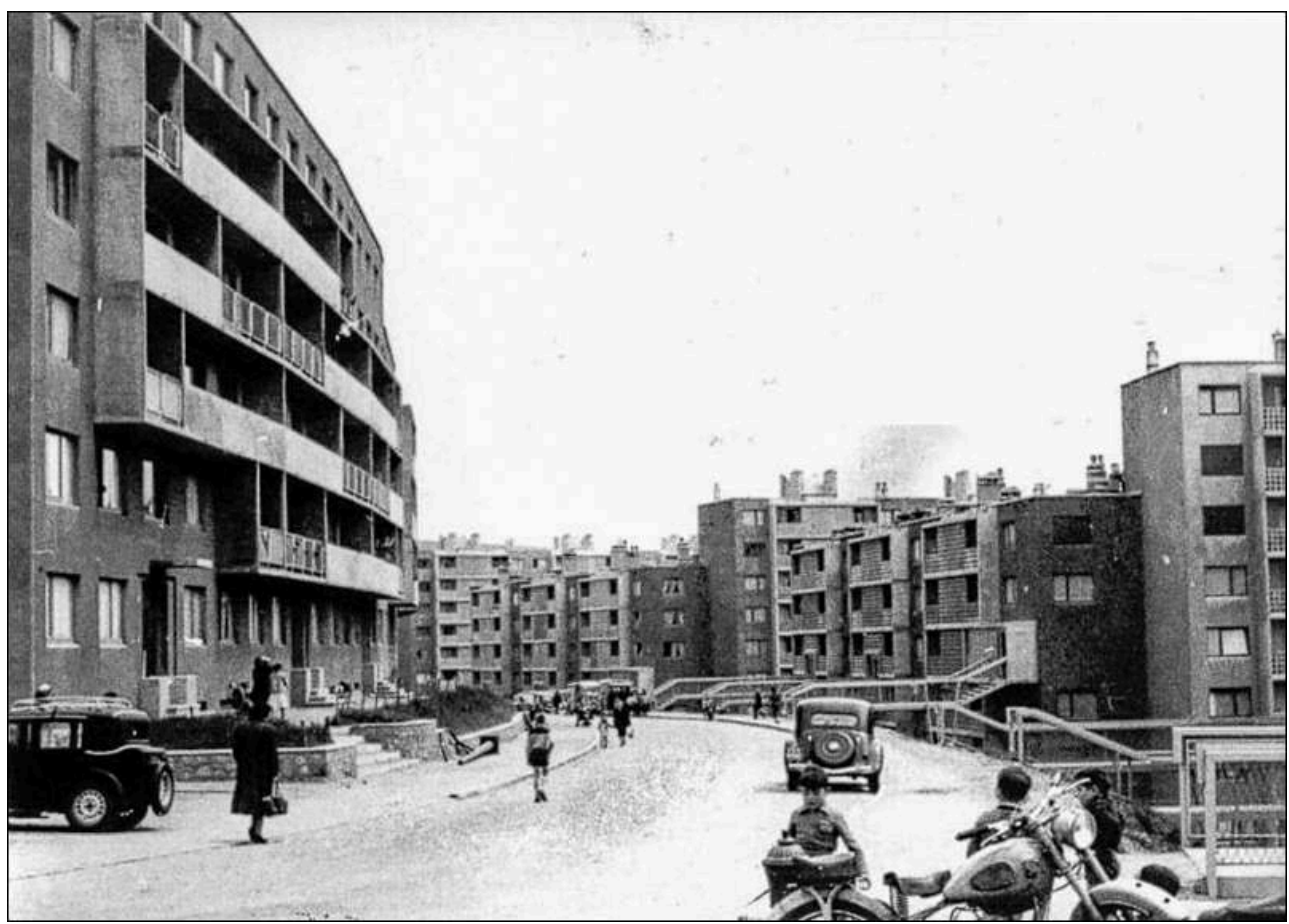

Source : Collection particulière.

Cette occupation sociale des grands ensembles du sud-est de Saint-Étienne dure jusque dans la deuxième moitié des années 1970, à partir de laquelle Montchovet voit sa population se paupériser, suivant des mécanismes connus (Dubet F., Lapeyronnie D., 1992 ; Blanc J.-N., Bonilla M., Tomas F., 2003) : les catégories les plus aisées commencent à quitter Montchovet dès le milieu des années 1970, la suite de leur parcours résidentiel les menant vers l'achat d'un nouveau logement (ailleurs dans la ville puis dans le périurbain qui se développe) ; une partie des ménages restant sur place se voit quant à elle petit à petit frappée par la crise socio-économique et le chômage qui touchent durement l'agglomération. Le cas stéphanois présente toutefois la particularité de connaître un processus exacerbé, avec une politique ethnique affichée sans détour. À partir du milieu des années 1970, Montchovet accueillent en effet quelques ménages d'origine étrangère, venant du logement spécifique ou des quartiers insalubres du centre-ville en voie de résorption ${ }^{4}$. Un choix s'impose alors aux gestionnaires de Montchovet (Ville et office): encourager le mouvement de paupérisation et d'« ethnicisation » ou le freiner5. La municipalité d'union de la gauche (1977-1983) tente de résister à ces évolutions en préparant avec les associations d'habitants une réhabilitation de Montchovet, retenue par l'État dans le cadre de la procédure Développement social des quartiers (DSQ, François Mitterand se rendant même luimême à Montchovet pour une visite express), visant à maintenir les catégories moyennes dans le grand ensemble ${ }^{6}$. Mais la municipalité RPR-UDF élue à la suite (1983) réoriente le projet, avec d'une part un volet bâti axé non plus principalement sur les logements mais sur une réhabilitation lourde des façades (isolation colorée, percées et démolition très partielle), et d'autre par un volet social expérimentant une gestion ségrégative de la ville. Montchovet se voit en effet attribuer une fonction d'accueil de familles d'origine maghrébine et aux revenus peu élevés. Le maire affirme : «au risque de choquer, je dirais que vouloir l'intégration à tout prix est un non-sens car personne ne la veut 
d'un côté comme de l'autre [...]. En revanche, je crois que vouloir faciliter les petits regroupements par ethnie serait préférable $»^{7}$. La paupérisation de la population de Montchovet va grandissant tout au long des années 1980 et 1990, avec pour corollaire les problèmes sociaux et de gestion qui en font un " quartier sensible », le plus dur de la ville: précarité ( $98,3 \%$ d'allocataires en 1996, taux de chômage de $34,3 \%$ ), échec scolaire, multiplication des actes d'incivilité et de délinquance (incendies nombreux de véhicules, agressions dont l'une tourne au drame), forte vacance de logements (68\% d'occupation totale en 1996)...

11 La dévalorisation de Montchovet est telle qu'en 1995, à peine plus de dix ans après la signature de la convention DSQ, le maire fraîchement élu (Parti radical), ex-adjoint à l'urbanisme du maire précédent, fait de la démolition de l'immeuble symbole du quartier la première grande décision de son mandat. Cette barre, le bâtiment $\mathrm{A}$ surnommé Muraille de Chine du fait de ses 250 mètres de long environ, de ses seize niveaux dans sa partie la plus haute et de ses 526 logements à sa livraison, est démolie le 27 mai 2000 (illustration 4).

Illustration 4 - Démolition de la Muraille de Chine

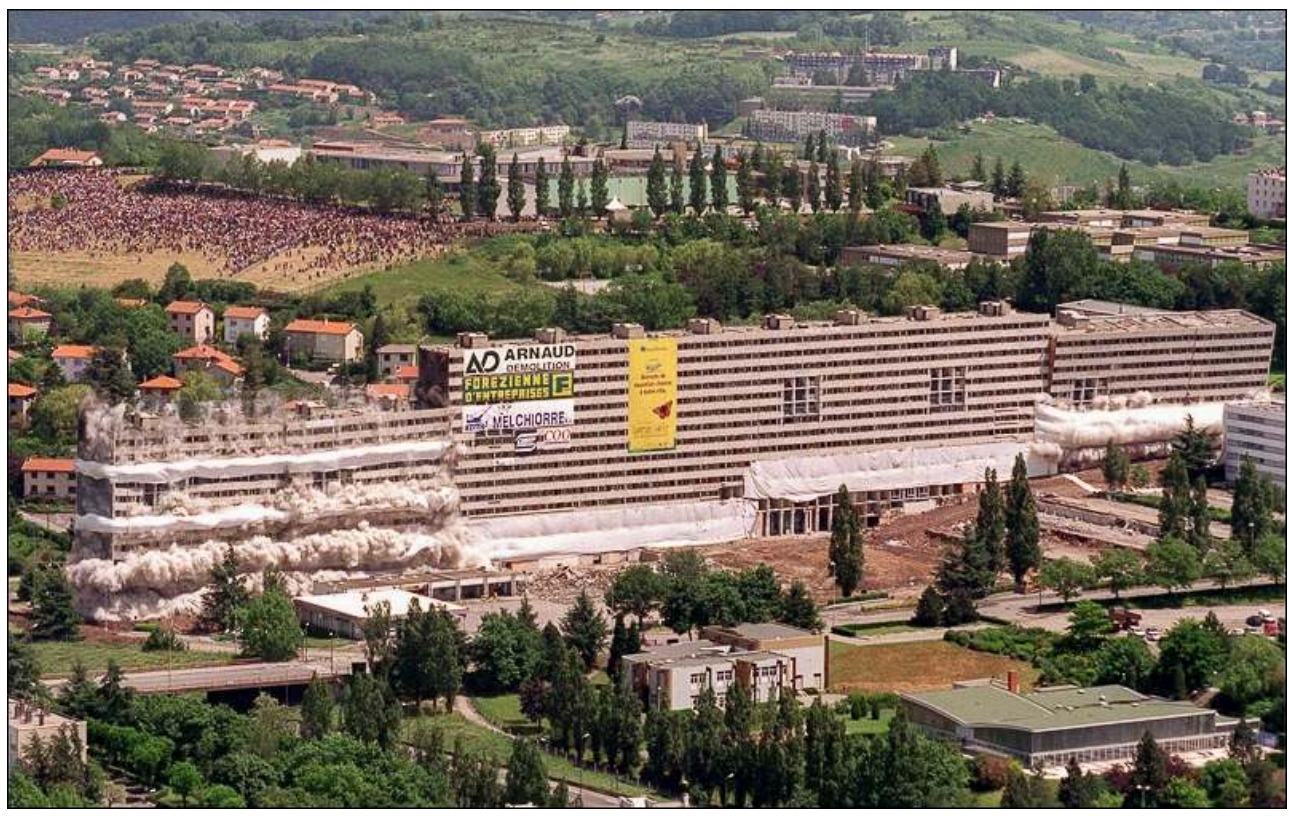

En haut à gauche, la foule est partagée, entre applaudissements des curieux et pleurs d'anciens locataires.

Source: Archives municipales de Saint-Étienne.

12 Le maire justifie cette démolition par un triple argumentaire : le rejet de l'immeuble par les Stéphanois (qu'il lit dans la vacance), la déconcentration des populations en difficulté (même si environ $70 \%$ des locataires sont in fine relogés dans le grand ensemble et ses voisins) et l'échec des réhabilitations menées jusqu'ici. L'évènement est fortement médiatisé, à l'échelle locale mais aussi nationale, puisque le ministre de la Ville Claude Bartolone vient à Montchovet tenir un discours signifiant que cette démolition par implosion, la première d'une série ayant lieu en 2000, marque un tournant dans le traitement des grands ensembles, avec passage à la rénovation urbaine. 
13 Pendant que Montchovet glisse dans les difficultés, le grand ensemble voisin de Beaulieu connaît une évolution discrète et tranquille, voyant simplement sa population vieillir mais garder un caractère assez mixte ${ }^{8}$. Et au moment même où la Muraille de Chine est démolie s'achève à Beaulieu une réhabilitation (façade, parties communes, implantation d'ascenseurs) qui donne toute satisfaction aux locataires si l'on en croit la presse $^{9}$ et le taux de vacance faible.

14 Ainsi, à l'entrée dans le nouveau millénaire et un demi-siècle après leur lancement, le contraste dans l'occupation sociale et les actions entreprises par les gestionnaires entre Beaulieu et Montchovet montre que les grands ensembles du sud-est de Saint-Étienne constituent des héritages alors dans des situations et des perspectives très variées. C'est dans ce contexte qu'au long des années 2000 des opérations de valorisation se développent tous azimuts dans et pour les grands ensembles stéphanois.

\section{Valorisation de patrimoine(s) dans les grands ensembles : processus polyphonique, voire cacophonique.}

Cette deuxième partie a pour objet d'inventorier et de présenter les différentes initiatives de valorisation de patrimoines dans les grands ensembles du sud-est de Saint-Etienne, plus finement que cela n'a été fait en introduction. Deux éléments qualifient cet inventaire. D'une part, la notion de patrimoine est ici prise dans ses différentes acceptions, afin de viser l'exhaustivité. D'autre part, une attention particulière est bien sûr portée aux démarches à l'initiative d'habitants, ou en impliquant. Des entretiens avec les instigateurs des différentes démarches ont été conduits afin de saisir leurs tenants et aboutissants.

La démolition de la Muraille de Chine ouvre la voie à un projet plus large encore. Soucieux d'enrayer le processus de dévalorisation de Montchovet, qui commence à s'étendre à certains immeubles des grands ensembles voisins (la Marandinière et la Palle), l'organisme d'HLM propriétaire, avec le soutien financier de la Ville, confie dès 2000 à l'urbaniste Loïc Josse la mission de définir un schéma directeur pour une intervention sur la totalité des grands ensembles HLM du sud-est (soit les grands ensembles HLM de Montchovet, Beaulieu, la Marandinière et la Palle). Les moyens de conduire cette intervention sur ce qui sera dénommé désormais les Quartiers sud-est sont trouvés d'abord dans un Grand projet de ville (2001), puis dans une convention ANRU (2005).

17 Dans le détail et pour la partie habitat ${ }^{10}$, les travaux mis en œuvre comprennent d'abord la démolition des plus grands immeubles (environ 500 logements, illustration 5), principalement à Montchovet (où il ne reste aujourd'hui plus qu'un sixième des logements initiaux) ; ensuite la reconstruction d'une douzaine d'immeubles de taille plus modeste, de statuts variés (HLM, locatif privé, propriété) et répondant à des demandes actuelles (développement durable, accès des logements sur l'extérieur avec des terrasses, etc.), ainsi que d'un lotissement de maisons mitoyennes; enfin la réhabilitation des immeubles conservés, avec entretien courant des appartements et parties communes, à Beaulieu notamment. 
Illustration 5 - Démolitions d'immeubles dans les grands ensembles du sud-est de Saint-Étienne

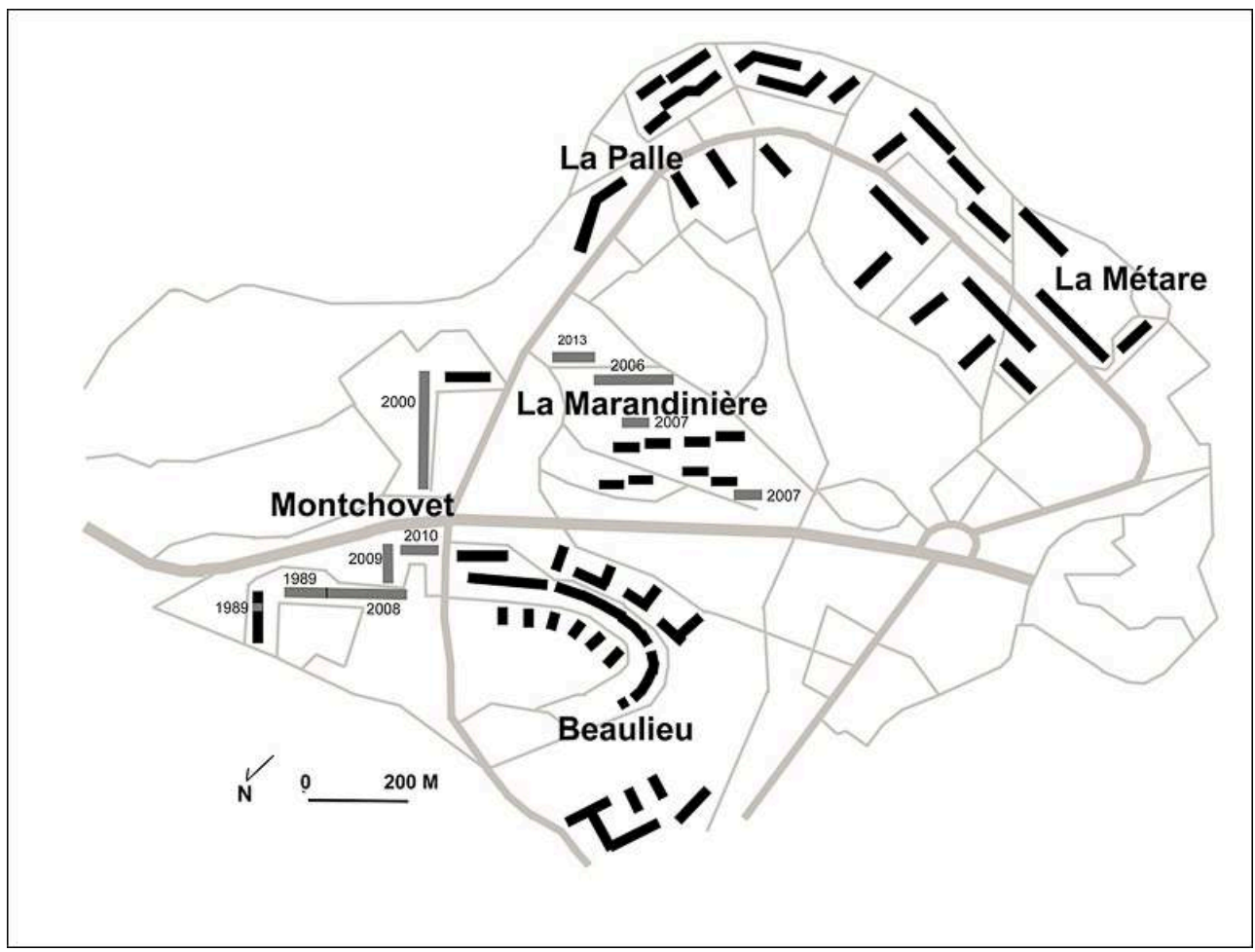

En gris, avec les dates.

Auteur: R. Kaddour.

18 La stratégie d'intervention sur le spatial est à ranger du côté de la communication, puisqu'il s'agit de changer l'image des grands ensembles via un remodelage profond. L'objectif, quant à lui, se situe du côté du peuplement, avec trois dimensions. Il s'agit d'abord de déconcentrer les familles en difficulté (Montchovet). Des efforts sont aussi déployés pour maintenir sur place les habitants les plus aisés restés à Beaulieu ou ailleurs, avec notamment des travaux d'adaptation des logements au vieillissement des populations. Il s'agit enfin de tenter d'attirer dans des logements neufs des populations de profils sociaux plus élevés que ceux d'une partie des familles en place, soit les plus aisés des demandeurs HLM en termes de revenus, ainsi que des catégories moyennes (dans les logements privés). En espérant ensuite qu'une osmose s'opère entre immeubles anciens et les immeubles nouveaux, et populations anciennes et populations nouvelles. Le programme stéphanois est de ce point de vue exemplaire de ce qui se passe dans d'autres grands ensembles en France (Stébé, 2010 ; Donzelot, 2012).

Ce programme est inscrit dans le Plan stratégique de patrimoine de l'organisme, soit un document qui, pour l'ensemble du parc, prévoit les interventions à mener dans le cadre d'une gestion pour les années voire les décennies à venir. Le terme de patrimoine est ainsi utilisé ici par l'organisme dans un sens immobilier et notarial : l'organisme fait in fine de la valorisation de patrimoine.

Mais si l'on prend le terme dans son sens culturel, d'autres opérations de valorisation de patrimoine(s) sont menées durant la même décennie, selon trois étapes. Celles-ci impliquent toutes, d'une façon ou d'une autre, des habitants.

21 Tout d'abord, l'annonce de la démolition de la Muraille de Chine engendre une série d'initiatives à ranger du côté du «travail de deuil, par lequel nous nous détachons des objets 
perdus de l'amour et de la haine ${ }^{11}$. Ce sont en premier lieu l'école primaire et le collège situés tout à côté de la Muraille qui, constatant le malaise suscité chez les enfants par la démolition à venir, conduisent un travail de photographie, de dessin et d'écriture afin de permettre de "mettre des mots sur des maux", les enseignants instigateurs ayant identifiés des "traumatismes" causés par l'annonce de la démolition ${ }^{12}$. Les dessins (illustration 6), poèmes et récits réalisés, publiés en 1999 dans le livre Murmures de Muraille $^{13}$ grâce aux moyens du Réseau d'éducation prioritaire, content principalement la douleur de voir l'immeuble disparaître, la crainte qu'inspire aux enfants la perte à venir des repères et habitudes, et une colère envers les décideurs engageant la démolition ${ }^{14}$.

\section{Illustration 6 - Dessin extrait de Murmures de Muraille}

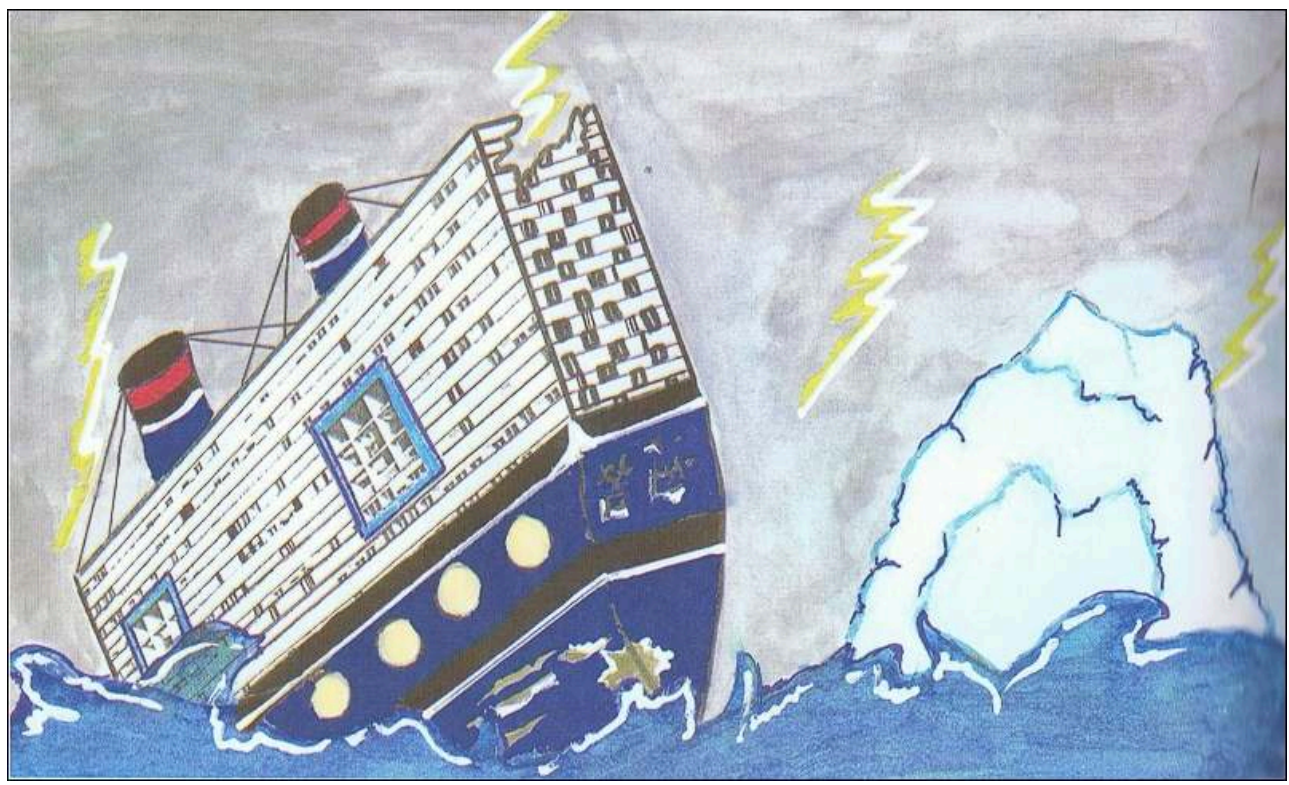

Source : École de Montchovet, collège Jean Dasté, 1999. Murmures de Muraille. Saint-Etienne, 91 p.

Quelques semaines avant la démolition de l'immeuble, c'est cette fois-ci avec des adultes qu'un travail similaire est mené, sans moyen, à l'initiative des associations et structures socioculturelles. Dans le cadre d'une "fête" de quartier, les désormais anciens locataires sont invités à présenter et partager leurs souvenirs, notamment en les inscrivant sur de larges feuilles apposées sur les murs de l'école primaire. Ces feuilles ont été depuis perdues, mais en 2008, un dispositif semblable est reconduit, à l'occasion de la démolition d'un autre immeuble dans le même secteur. Aux dires des habitants présents lors des deux manifestations, les propos formulés sont sensiblement les mêmes. Ils sont cette fois-ci consignés par le centre social et reproduit dans un petit dossier papier 14 juin 2008, rue Pierre-Loti, 40 ans de souvenirs. Ils portent surtout sur le vivre-ensemble dans ce grand ensemble depuis l'uniformisation de la population, avec des récits de faits, pour certains anecdotiques, jugés positifs (la découverte du confort dans ces immeubles ${ }^{15}$, l'entraide entre voisins amis, les jeux d'enfants, les festivités et rites musulmans ${ }^{16}$ ) ou négatifs (la délinquance, le sentiment d'abandon par les pouvoirs publics, le traumatisme de la rénovation urbaine dans le quartier ${ }^{17}$ ). En somme, comme dans le cas de Murmures de Muraille, ces témoignages consignés et diffusés sont des éléments visibles d'une mémoire partagée d'un vivre-ensemble (communautaire), et d'une mémoire de difficultés quotidiennes et de luttes. Ces mémoires constituent un 
patrimoine immatériel. Enfin, quelques années après la démolition de l'immeuble, la mémoire d'anciens locataires est à nouveau sollicitée lorsque l'Opéra-Théâtre de la Ville de Saint-Étienne, qui dispose d'une Unité de production audiovisuelle, prend l'initiative de réaliser un film documentaire de 32 minutes sur "la réussite de la construction, l'échec de la restauration ${ }^{18}$ et les raisons de la démolition de la Muraille de Chine ${ }^{19}$. L'équipe est animée par une double volonté de "garder une trace, puisque c'était un immeuble très singulier de Saint-Étienne » et de «traiter du ressenti de cette perte chez ses habitants $\aleph^{20}$. Le film prend le parti de s'appuyer très majoritairement sur des interviews. Les témoins sont toutefois principalement des locataires de la première heure ${ }^{21}$, si bien que ce sont donc surtout les premières années qui sont contées, avec la modernité de l'immeuble et la découverte du confort. Une place est toutefois laissée aussi à des récits de la lutte pour l'équipement du quartier (à sa livraison, pendant le DSQ) et contre sa relégation (pendant les années les plus difficiles).

23 Ensuite, après ce travail de deuil, de recueil de témoignages et de valorisation de mémoires, une autre forme de valorisation de patrimoine culturel survient en 2003, avec la labellisation Patrimoine du XXe siècle de Beaulieu par le ministère de la Culture et de la Communication, en raison de ses spécificités bâties (forme organique de la composition, adaptation au relief, travail sur les parcs et jardins) et historiques (caractère pionnier, apport au quotidien domestique). Celles-ci sont identifiées depuis la fin des années 1980 par des travaux de l'École d'architecture et de l'Université de Saint-Étienne.

Enfin, deux ans après, à l'occasion du cinquantenaire du grand ensemble (2005), SaintÉtienne Ville d'art et d'histoire ${ }^{22}$, chargé de la valorisation et l'animation du patrimoine stéphanois, réalise un projet intitulé Beaulieu, patrimoine urbain $d u X^{e}$ siècle, comprenant, outre la pose de la plaque-label, la pose d'une signalétique patrimoniale, l'édition d'un ouvrage sur l'histoire et les spécificités bâties du site et la reconstitution d'un appartement des années 1950 proposé à la visite. Ce dernier est réalisé avec l'aide d'habitants installés depuis l'origine : ils donnent ou prêtent la quasi-totalité des objets exposés, et font part de souvenirs qui permettent d'en concevoir le discours de visite. Celui-ci porte sur la découverte du confort moderne permise par les grands ensembles, sur le quotidien domestique dans les années 1950 (activités féminines, masculines, des enfants), mais également sur l'architecture et l'urbanisme des Trente glorieuses. L'école primaire de Beaulieu participe quant à elle à ce cinquantenaire, avec la réalisation d'un petit film documentaire et d'animation sur la naissance du quartier. Le propos porte sur la crise du logement et la forme bâtie originale de Beaulieu.

Ainsi, le lien établi entre les différentes acceptions du terme de patrimoine (culturelle et notariale-immobilière) ${ }^{23}$ permet de conforter le constat fait en introduction d'une «patrimonialisation » des grands ensembles du sud-est de Saint-Étienne. Ce processus de "patrimonialisation" est complexe, car polyphonique: les nombreuses actions croisées, émanent d'acteurs très variés, de la puissance publique (État, Ville) aux associations d'habitants, en passant par des institutions comme l'organisme d'HLM, les écoles ou les équipements socioculturels de quartier. Mais le processus est aussi assez cacophonique. En effet, pour chacun de ces acteurs, les motivations sont variables et le patrimoine à valoriser ne recouvre pas les mêmes éléments. Ces éléments peuvent même être contradictoires voire hermétiques les uns par rapport aux autres. Par exemple, les mémoires des premiers locataires ayant connu la mixité sociale diffèrent de celles des derniers locataires de Montchovet ayant connu le "ghetto ». De même, les 
locataires sont globalement peu sensibles au patrimoine architectural tel que le valorisent la DRAC et Saint-Étienne Ville d'art et d'histoire (si l'on en croit le profil des visiteurs - majoritairement des Stéphanois d'autres quartiers, et des scolaires).

La complexité du processus global de "patrimonialisation » comme les contradictions entre les démarches), n'est pas problématique en elle-même, et tendrait plutôt à être une richesse. Mais elle ne permet pas de comprendre au premier abord les tenants et aboutissants de la " patrimonialisation ». C'est donc à l'identification de ceux-ci que les lignes suivantes vont s'attacher, dans le but de mieux cerner la place et le rôle que les démarches habitantes tiennent et jouent dans la patrimonialisation.

\section{Récupération, instrumentalisation et délaissement des paroles des locataires : mémoire du mouvement HLM contre mémoires d'habitants}

27 La contextualisation historique, la présentation des acteurs et enjeux des démarches de valorisation de patrimoines et le recueil de matériaux réalisés permettent à présent, dans cette dernière partie, de donner du sens à cette association cacophonique entre grand ensemble et patrimoine, en en fournissant des clés d'explication. L'image peut être celle d'un "système » complexe (la " patrimonialisation »), dont les composantes (acteurs, motivations, initiatives) ont été démontées plus haut, et qu'il s'agit à présent de remonter pour en comprendre la logique d'ensemble. Puisqu'il s'agit d'un processus global de (re)valorisation, donc d'un bénéfice espéré, on peut se demander à qui profitent la "patrimonialisation » et les différentes démarches de valorisation qui la compose, dans leur cohérence d'ensemble éventuelle? La réflexion doit porter en particulier sur la «patrimonialisation" de Beaulieu, puisque Montchovet n'existe presque plus.

Dans une logique chère à la géographie sociale, celle du « renversement de l'ordre des facteurs" (Rochefort, 1982), c'est plus particulièrement autour de la question du peuplement (Morel-Journel, Sala-Pala, 2011) qu'une interprétation va être ici proposée, puisque, comme montré précedement, cette question est au cœur de l'histoire et de l'actualité des grands ensembles, et donc au cœur de leur « patrimonialisation ».

La "patrimonialisation" des ensembles de logements sociaux est généralement associée à des initiatives de préservation du bâti, principalement en raison de son exemplarité architecturale ${ }^{24}$. Mais ces initiatives apparaissent souvent aux organismes d'HLM comme discordantes avec les logiques de gestion, en particulier l'adaptation du bâti à la vie qui continue (évolutions sociales, vieillissement du cadre physique, etc.). Des cas nombreux, en région parisienne notamment ${ }^{25}$, montrent que les organismes ne souhaitent en effet généralement pas voir leurs immeubles mis "sous cloche ${ }^{26}$. Toutefois, cette réticence à la «muséfication» ne signifie pas insensibilité à l'architecture et aux spécificités du bâti. Au contraire, dans le cas de Beaulieu à SaintÉtienne, l'organisme paye même la pose de la plaque-label Patrimoine du XXe siècle (label sans incidence juridique ni exigence de restauration), et affirme avoir tenu à " faire des greffes qui vont dans le sens initial » lors des réhabilitations de 2000 et 2006 (matériaux, couleurs et modénatures des façades et des ascenseurs et passerelles construits, références aux années 1950 dans les fresques peintes). Mais ce respect de l'architecture moderne tient plus au fait que cette dernière a été l'outil, le signe et la matérialisation 
d'un projet sociopolitique lui-même moderne. Via ce que l'on nomme le «mouvement HLM », il s'agissait d'offrir un logement décent et confortable au plus grand nombre. Beaulieu, groupe HLM parmi les plus anciens produits sur le territoire stéphanois, est pour l'organisme propriétaire un symbole de l'institution et de ses missions. Occupé aujourd'hui encore par une population relativement mixte, il représente plus précisément un emblème et la mémoire de l'idée initiale d'un habitat social pour tous, et non pour les seuls démunis ${ }^{27}$ comme a pu le devenir Montchovet par exemple.

Mais la démarche de valorisation des spécificités de Beaulieu par l'organisme va plus loin encore. D'une part son architecture adaptée à la colline sert de modèle pour la reconstruction (en cours) du grand ensemble de la Marandinière (200 logements) situé sur la colline voisine (jeu de symétrie inversée, illustration 7), et d'autre part une imitation de sa relative mixité sociale est tentée avec le découpage de cette nouvelle opération en statuts variés (locatif privé et public, accession sociale à la propriété). Beaulieu est ainsi un point d'appui, y compris donc au sens topographique, pour la « reconquête» du reste du parc de grands ensembles qui a connu une paupérisation de sa population. Ce grand ensemble est ainsi pour l'organisme et la Ville un modèle refondateur pour les Quartiers sud-est remodelés et reconstruits.

Illustration 7 - Beaulieu et la nouvelle Marandinière face à face

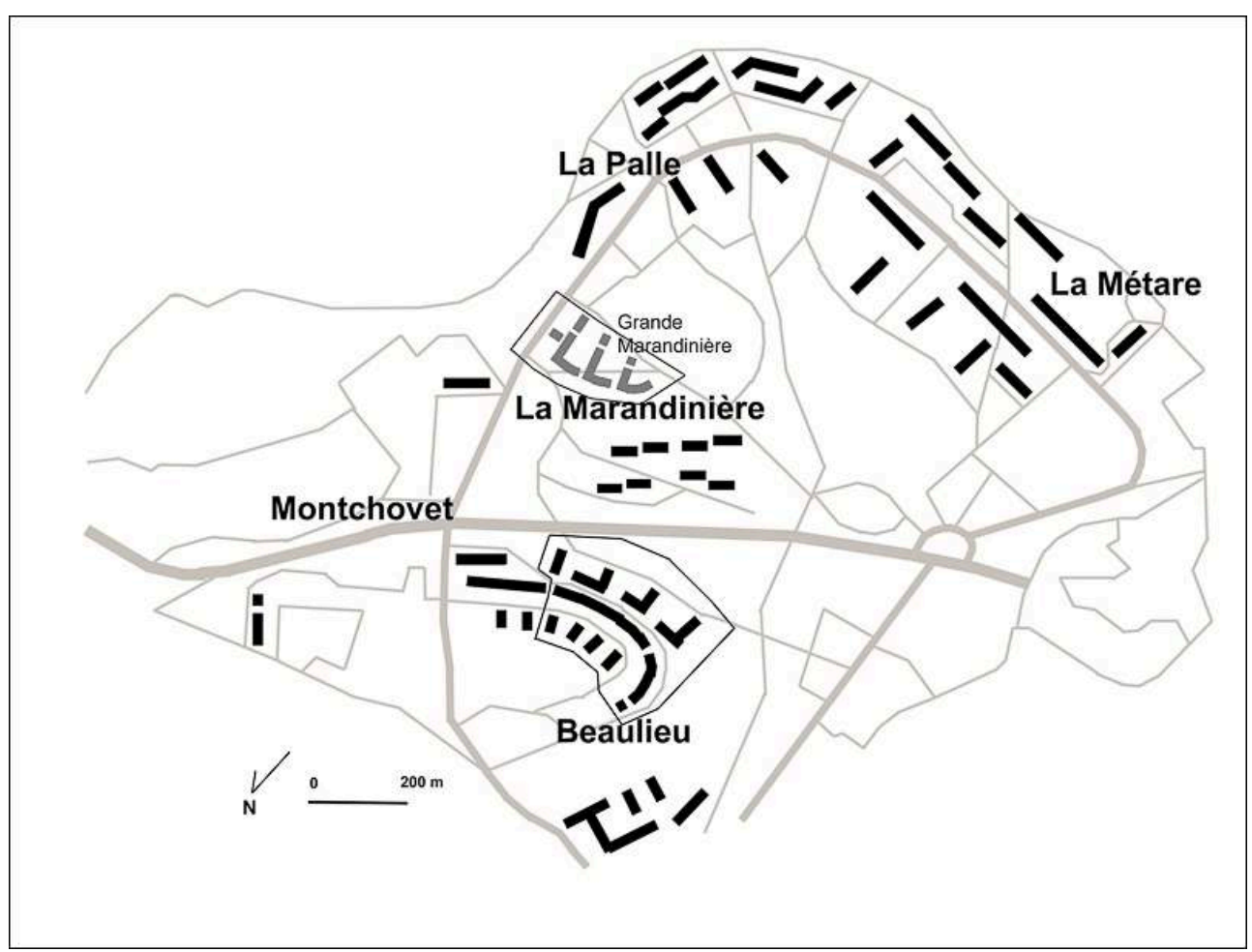

Située au sommet de la colline voisine, l'opération de reconstruction de la Marandinière (en gris) répond à Beaulieu, en un jeu de symétrie inversée.

Auteur : R. Kaddour.

En somme, un retour aux origines du mouvement HLM est tenté. Ce sont ce rapport au passé et cette sollicitation d'une « identité » du mouvement HLM qui donnent du corps à l'idée de patrimoine. Ainsi replacée dans la perspective des logiques de gestion par l'organisme HLM et ses partenaires institutionnels, la "patrimonialisation» des Quartiers sud-est apparaît donc à la fois comme l'expression des logiques qui ont porté 
la production de logements sociaux, et le levier de mise en cohérence de stratégies de réhabilitation matérielle et symbolique des grands ensembles du sud-est.

Cette démarche de construction et de valorisation d'un patrimoine et d'une mémoire du mouvement HLM se développe contre la construction et la valorisation d'autres patrimoines et mémoires, notamment ceux des habitants. Le terme de « contre » est ici à prendre dans ses deux sens, à commencer par celui de proximité. En effet, cette "patrimonialisation" peut s'alimenter des démarches valorisant les témoignages et mémoires des plus anciens locataires, ceux ayant connu les premières heures des grands ensembles de Beaulieu et Montchovet et l'occupation initiale mixte à laquelle les gestionnaires tentent de revenir. De ce fait, l'organisme est partenaire de toutes les actions renvoyant au contexte de construction de Beaulieu et aux années de sa jeunesse (comme de celle des autres grands ensembles). L'appartement reconstitué des années 1950 avec son mobilier de catégories ouvrières et moyennes et son discours de visite signalant en creux l'occupation mixte initiale en est une bonne illustration: l'organisme met l'appartement à disposition et paye les travaux pour les murs et sols. D'autres actions allant dans le même sens comme l'ouvrage ou les films sur la construction de Beaulieu reçoivent un soutien bienveillant de l'organisme d'HLM. Il est possible de parler ici de récupération, voire en quelque sorte d'instrumentalisation.

Le partenariat avec l'organisme, ou son soutien, permettent aux témoignages et mémoires d'habitants de la première heure (principalement locataires de Beaulieu et catégories moyennes et ouvrières) d'être recueillis et valorisés dans des supports soignés, durables et réalisés avec des moyens significatifs : des films, un ouvrage et l'appartement reconstitué des années 1950. Ce qui n'est pas le cas d'autres témoignages et mémoires d'habitants, en particulier ceux des locataires les plus pauvres et arrivés récemment. Car la démarche de valorisation d'une mémoire du mouvement HLM se développe en effet contre les mémoires d'habitants, c'est-à-dire aussi en opposition à ces dernières. Les mémoires qui ne renvoient pas à une occupation mixte ne bénéficient pas ou très peu du soutien de l'organisme d'HLM ou de la Ville, puisque le discours qu'elles produisent est discordant par rapport au discours institutionnel. Les récits de vie communautaire et dans la précarité d'habitants de Montchovet devenu "ghetto ", et plus encore les récits de lutte face aux institutions pour l'équipement du quartier ou contre les démolitions, s'expriment alors dans des supports éphémères et réalisés sans moyen : prise de parole lors de "fêtes", impression de paroles sur support papier simple diffusées dans un cercle très restreint. Ces mémoires ne sont alors posées et exposées dans l'espace et le débat publics que de manière très discrète, ce qui ne peut les faire accéder à un statut de patrimoine pour d'autres acteurs que les seuls (ex)locataires concernés.

Pour conclure, ce cas stéphanois rappelle que la "patrimonialisation " générale constatée en France touche aussi les marges de la ville. Le mouvement n'est pas neuf et a déjà été analysé pour la cité Tony Garnier à Lyon $^{28}$ ou Firminy-Vert ${ }^{29}$ (Loire) par exemple. Chacun de ces cas montre à quel point le processus est complexe, avec notamment des acteurs nombreux (gestionnaires, institutions culturelles, habitants plus - Lyon - ou moins - Firminy - moteurs ou considérés...) et des motivations et finalités variées (culturelles, économiques, symboliques, etc.). Saint-Étienne n'échappe pas à cette complexité. Il a donc d'abord fallu s'attacher ici à trouver du sens à la démarche d'ensemble menée dans les Quartiers sud-est. L'analyse dans l'épaisseur historique a permis d'expliciter une entreprise globale portée par l'organisme 
propriétaire : Beaulieu, emblème de l'idée initiale d'un habitat social pour tous (et non pour les seuls démunis), est consolidé et imposé comme modèle pour la réhabilitation, dans ses aspects matériel et juridique, des grands ensembles paupérisés. C'est cette recherche de l'état initial qui donne le plus de sens à la "patrimonialisation ", processus de valorisation tant symbolique que matériel.

Une fois ce préalable posé, l'analyse a permis de montrer que cette démarche d'ensemble fait une place inégale aux différentes mémoires : celles des plus anciens locataires, aux profils ouvriers ou de catégories moyennes, sont recueillies et valorisées, alors que celles des habitants les plus précaires sont délaissées. Ainsi, les catégories d'habitants les plus populaires des Quartiers sud-est (pour partie significative d'origine maghrébine), fragilisées par la précarité, contraintes de faire face au relogement (et donc implicitement à la déconsidération de leur habitat par les institutions gestionnaires et l'opinion publique), voient aussi leur place dans la société questionnée dans le domaine symbolique par la thématique patrimoniale. Ces catégories sociales ne laissent pas ou peu de traces, alors que le champ mémoriel et patrimonial est pourtant bel et bien ouvert dans les quartiers populaires. Ce constat formulé à partir du cas stéphanois est partagé par les sociologues et géographes développant des analyses et perspectives de réflexion autour du droit au patrimoine, du caractère socialement sélectif de la construction du patrimoine et de la participation de ce dernier aux systèmes des inégalités (Veschambre, 2007, 2008; Pinçon-Charlot, Pinçon, 2007).

\section{BIBLIOGRAPHY}

Blanc J-N., Bonilla M., Tomas F., 2003. Les grands ensembles, une histoire qui continue. Saint-Étienne, Publications de l'Université de Saint-Étienne, $260 \mathrm{p}$.

Donzelot J. (dir.), 2012. A quoi sert la rénovation urbaine. Paris, PUF, 237 p.

Dubet F., Lapeyronnie D., 1992. Les quartiers d'exil. Paris, Le Seuil, 251 p.

Gravari-Barbas M., 2002. Le patrimoine territorial. Construction patrimoniale, construction territoriale : vers une gouvernance patrimoniale? Angers, Cahiers de l'ESO.

Hertzog A., 2011. Les géographes et le patrimoine. EchoGéo [En ligne], 18 | 2011, mis en ligne le 05 décembre 2011. URL : http://echogeo.revues.org/12840 ; DOI : 10.4000/echogeo.12840

Kaddour R., 2013. Quand le grand ensemble devient patrimoine : réflexion à partir de cas exemplaires en région stéphanoise. Thèse de doctorat de géographie, Université Jean Monnet de Saint-Etienne.

Morel Journel C., Sala Pala V., 2011. Le peuplement, catégorie montante des politiques urbaines néolibérales? Métropoles [En ligne], 10. URL : http://metropoles.revues.org/4536

Pinçon M., Pinçon-Charlot M., 2007. Les ghettos du Gotha. Comment la bourgeoisie défend ses espaces. Paris, Le Seuil, 294 p. 
Pouvreau B., 2011. Faut-il 'patrimonialiser' les grands ensembles? Métropolitiques, URL : http:// www.metropolitiques.eu/Faut-il-patrimonialiser-les-grands.html

Ricoeur P., 2000. La mémoire, l'histoire, l'oubli. Paris, Le Seuil, 689 p.

Rochefort R., 1982. Réflexions liminaires sur la géographie sociale. In D. Noin (Coord.), Actes du colloque de géographie sociale, Lyon, 1982, p. 11-15.

Stébé J.-M., 2010. La crise des banlieues. Paris, Presses universitaires de France, 127 p.

Thomas E., 1992. Politiques urbaines et transformations socio-spatiales, l'exemple stéphanois 1977-1991.

Thèse de doctorat de géographie, Université de Saint-Étienne, 438 p.

Vant A., 1974. Les grands ensembles du sud-est de Saint-Etienne : essai de géographie sociale. Saint-

Étienne, CIER-SR, dossier $n^{\circ} 9,78 \mathrm{p}$.

Veschambre V., 2002. Une mémoire urbaine socialement sélective, réflexions à partir de l'exemple d'Angers. Les Annales de la recherche urbaine, $n^{\circ}$ 92, p. 65-74.

Veschambre V., 2007. Patrimoine : un objet révélateur des évolutions de la géographie et de sa place dans les sciences sociales. Les annales de géographie, $n^{\circ}$ 656, Paris, Armand Colin, p. 361-381.

Veschambre V., 2008. Traces et mémoires urbaines. Rennes, Presses Universitaires de Rennes, 315 p.

\section{NOTES}

1. L'expression est tirée du film La crise du logement, 1954, réalisation Roger Montéran, Maryse Barbut et Geneviève Cortier.

2. Les cinq autres sont: Angers (677 logements), Boulogne-Billancourt (800 logements), BronParilly (2 600 logements), Le Havre (1 400 logements) et Pantin (800 ogements).

3. Montchovet présente simplement une population plus jeune, du fait de plus grands appartements ; il accueille d'ailleurs à sa livraison les familles agrandies de Beaulieu.

4. En 1979, $46 \%$ des nouveaux arrivants sont étrangers. Les chefs de ménages étrangers représentent à cette date 40 \% des chefs de ménages de Montchovet, contre moins de $3 \%$ en 1972 (Thomas, 1992).

5. L'acuité du questionnement local se voit par ailleurs accru par le contexte national puisque, parallèlement, la réforme du logement de 1977 et ses effets, notamment ceux de l'Aide personnelle au logement, participent à paupériser la population du logement social en général et des grands ensembles réhabilités en particulier : l'augmentation des loyers liée aux travaux est, dans le budget des familles, absorbée par l'APL pour les ménages qui y ont droit, mais fait de fait fuir les familles les plus aisées qui ne peuvent y prétendre (Blanc J-N., Bonilla M., et Tomas F., 2003).

6. "L'office doit conserver un caractère social mais néanmoins, il ne peut pas accueillir n'importe qui et se transformer en cité de l'Abbé Pierre ou en asile de nuit », affirme notamment l'élu président de l'office HLM. Compte-rendu du CA de l'office, 3 janvier 1979.

7. «Quand la Muraille de Chine s'éveillera », Hebdo, 2 novembre 1983.

8. Affirmation basée sur des entretiens avec le personnel de l'organisme d'hlm, les responsables de l'Amicale des locataires, mais aussi des observations et une pratique du terrain. Les statistiques n'ayant pas pu être obtenues auprès de l'organisme.

9. «Beaulieu - le Rond-Point. Une importante opération de réhabilitation», La Tribune-Le Progrès, 15 décembre 1999.

10. Le schéma prévoit aussi l'implantation d'équipements privés (zone d'activité) et publics (piscine municipale, espaces publics...). 
11. Ricoeur P., 2000. La mémoire, l'histoire, l'oubli. Paris, Le Seuil, p. 577.

12. Passages en italiques extraits d'un entretien avec l'ex-directeur de l'école primaire de Montchovet, 14 janvier 2009.

13. École Montchovet et collège Jean-Dasté Saint-Etienne, 1999. Murmures de Muraille. Imprimerie Sud-Offset, La Ricamarie, $91 \mathrm{p}$.

14. Les textes sont trop longs pour être reproduits ici, et il est difficile d'en extraire des parties sans leur enlever leur cohérence. Ce poème donne toutefois un aperçu : «La Muraille va exploser, Il va y avoir de la fumée, Les gens vont tous déménager, La Muraille va se vider. Il n'y aura plus les cris des enfants, Qui sont tous les jours accompagnés par leur maman. Le marché ne sera plus rempli par les clients. Que va alors devenir le quartier maintenant, Sans les parents et leurs enfants? La Muraille va exploser, Vat-on laisser la moitié ? Ou le quart de la moitié ? C'est à vous de le demander. Car c'est vous qui y habitez. Qu'envisagez-vous de faire Pour que le quartier puisse vous plaire? Il ne faut surtout pas se taire Car Montchovet nous est cher ».

15. « En 1971, [...] dans ce quartier, j'habitais un appartement de 5 pièces, plus la cuisine; un logement que j'ai qualifié d'emblée comme l'un des meilleurs de Saint-Étienne. Les pièces étaient confortables, lumineuses, grandes et propres ".

16. "Je me souviens du lieu de prière avec les hommes tous en djellabas blanches, qui se pressaient pour rentrer chez eux afin de prendre le repas du soir. Qu'après la rupture du jeûne, les pères se retrouvaient dans un local du gymnase Georges-Puillet autour d'un café ou d'un thé pour passer la soirée. Que pour la fête de l'Aïd, on se levait avec le soleil pour aller faire la prière de ce jour de fête au lieu de culte. Chacun arrivant avec des friandises diverses qui étaient ensuite partagées entre les participants, sans oublier les enfants qui étaient à l'extérieur».

17. "C'est de la rancour et de la haine que j'éprouve en écrivant ces quelques lignes. Montchovet restera une légende gravée dans mon cœur ».

18. Présentation du film sur la pochette du DVD.

19. 27 mai 2000, 13h : démolition d'un immeuble moderne baptisé Muraille de Chine, 2002, réalisation Jean-Claude Parayre, Esplanade Opéra-Théâtre de Saint-Etienne, 32 minutes.

20. Entretien avec l'ex-directeur de l'image du film, 16 février 2010.

21. Le maire refuse d'être interviewé.

22. Direction des Affaires culturelles de la Ville de Saint-Étienne.

23. Une plaquette d'information produite par la Ville (n.d.) associe d'ailleurs ces deux aspects : à côté d'images de travaux et de dessins des nouvelles constructions figure un encadré sur la labellisation Patrimoine du XXe siècle de Beaulieu.

24. Lire à ce sujet : Collectif, 2010. 1945-1975. Une histoire de l'habitat. 40 ensembles " Patrimoine du XXe siècle ». Paris, Beaux arts éditions, 96 p. Comité des Grands prix nationaux de l'architecture, 2008. Faut-il protéger les grands ensembles? Rapport de la Direction de l'Architecture et du Patrimoine, $125 \mathrm{p}$.

25. Etoiles de Jean Renaudie à Villetaneuse, Serpentin d'Emile Aillaud à Pantin, cité de l'Etoile de Georges Candilis à Bobigny.

26. Toutes les citations de ce paragraphe sont des propos du directeur de l'organisme d'HLM propriétaire de Beaulieu.

27. Ce qui, l'histoire le montre, peut être un effet de certaines politiques et lois.

28. Foret C., 1993, Valorisation et dévalorisation dans la ville : le musée urbain Tony Garnier ou la ré-invention d'une cité HLM, Rapport pour leministère de l'Equipement et du Logement, $186 \mathrm{p}$.

29. GAY G., 2008, "De Firminy-Vert à Le Corbusier Ville: la construction d'un patrimoine paradoxal », dans: GUILlot Xavier (dir.), Firminy, Le Corbusier en héritage, Saint-Etienne, Publications de l'Université de Saint-Etienne, pp. 133-153. Veschambre V., 2000, " Firminy-Vert et le 'site Le Corbusier' : vers une esthétisation du grand ensemble?», Cahiers de la Méditerranée, $n^{\circ} 60$, juin, pp. 133-156. 


\section{ABSTRACTS}

The research examines Montchovet and Beaulieu, two of the four contrasting social housing estates composing southeastern Saint-Etienne (France). A local plan carried out in this place, with two aspects. First, it provides the demolition of Montchovet because of its pauperization during the 80 s. Second, Beaulieu (socialy mixed) become established as model that can redefine the other social housing estates. These operation is emblematic of a progressive return to the roots and a heritage process. But this project also neglects and erases the histories of the poor, the workers and the immigrants.

La réflexion porte sur Montchovet et Beaulieu, deux des quatre grands ensembles contrastés du sud-est de Saint-Étienne. Un schéma directeur d'ensemble y est mis en œuvre en deux volets. Il prévoit d'une part la démolition de Montchovet suite à sa paupérisation au long des années 1980. Il fait d'autre part de Beaulieu, encore socialement mixte, un emblème d'une certaine idée initiale de l'habitat social pour tous, mais aussi un modèle pour une réhabilitation, aux sens matériel et juridique du terme, des autres grands ensembles. Ce retour aux sources participe d'une «patrimonialisation». Mais celle-ci fait une place inégale aux différentes mémoires d'habitants : celles des plus anciens locataires, aux profils ouvriers ou de catégories moyennes, sont récupérées et valorisées, alors que celles des habitants les plus précaires sont délaissées.

\section{INDEX}

Mots-clés: grand ensemble, rénovation urbaine, patrimoine, patrimonialisation, mémoire, participation habitante, inégalité sociale, Saint-Étienne

Keywords: Social housing estates, urban renewal, heritage, heritage process, memory, participation of residents, social inequality

Subjects: Sur le Champ - Sur le Terrain

\section{AUTHOR}

\section{RACHID KADDOUR}

Rachid Kaddour, rachid.kaddour@st-etienne.archi.fr, est Géographe, maître assistant associé, membre de l'Unité de recherche en formation Transformation(s), École nationale supérieure d'architecture de Saint-Etienne. Il a publié récemment :

- Kaddour R. (en cours). L'imaginaire démolisseur observé dans les récits institutionnels : l'exemple de la rénovation urbaine à Saint-Etienne (1995-2011). In Fijalkow Y. (dir.), Dire la ville c'est faire la ville, Presses universitaires de Rennes, Rennes.

- Kaddour R., Juan A., 2015. Histoire d'un immeuble démoli dans un grand ensemble rénové : la tour Plein-ciel (Saint-Etienne), représentations en trois actes. In Jurado P., Callens A.-C. (dir.), Art, architecture, paysage à l'ère postindustrielle, PUSE, Saint-Etienne.

- Par ailleurs, la thèse de doctorat de R. Kaddour sur la patrimonialisation des grands ensembles est en voie de publication (sortie prévue en 2016). 